\title{
Die Verfassungsmäßigkeit der sog. 10-H-Regelung in Art. 82 BayBO: Eine Neubewertung nach dem Klima-Beschluss des BVerfG*
}

\author{
Kurt Faßbender
}

(C) Der/die Autor(en) 2021. Dieser Artikel ist eine Open-Access-Publikation.

Gegenstand des nachfolgenden Beitrags ist die Frage der Verfassungsmäßigkeit der sog. 10-H-Regelung in Art. 82 der Bayerischen Bauordnung (BayBO). Dabei weist das Gutachten nach, dass diese - bislang in der Rechtsprechung nur vom Bayerischen Verfassungsgerichtshof aus der Perspektive der Verfassung des Freistaates Bayern näher geprüfte - Frage nach dem sog. Klima-Beschluss des BVerfG vom 24.3.2021 neu bewertet werden muss.

\section{Einführung}

Jede Person, die sich fachlich fundiert mit dem Ausbau der Windenergie an Land befasst, ist sich darüber im Klaren, dass die Flächenverfügbarkeit hier das größte Problem darstellt. Dessen ungeachtet enthält die Bayerische Bauordnung seit dem Jahr 2014 eine Regelung, die dazu führt, dass Windenergieanlagen in Bayern grundsätzlich nur noch dann gebaut werden dürfen, wenn der Abstand zur nächstgelegenen Wohnbebauung mindestens dem Zehnfachen der Anlagenhöhe $(\mathrm{H})$ entspricht. Dementsprechend muss der Abstand zur nächstgelegenen Wohnbebauung bei modernen Windenergieanlage, die meist eine Höhe von $200 \mathrm{~m}$ oder mehr aufweisen, mindestens $2000 \mathrm{~m}$, also mindestens $2 \mathrm{~km}$ betragen. Diese sog. 10-H-Regelung hat, wie im Folgenden noch darzulegen sein wird, schnell dazu geführt, dass der Ausbau der Windenergie in Bayern im Einklang mit dahingehenden Prognosen dauerhaft eingebrochen ist.

Angesichts dessen war die 10-H-Regelung von Anfang nicht nur (umwelt-)politisch, sondern auch verfassungsrechtlich umstritten. Dessen ungeachtet hat der Bayerische Verfassungsgerichtshof (BayVerfGH) die 10-H-Regelung in seiner Entscheidung vom 9.5.2016 aus der Perspektive der Verfassung des Freistaates Bayern im Wesentlichen gebilligt. Daher wird die 10-H-Regelung (weiterhin) angewendet; weitere Initiativen mit dem Ziel einer verfassungsgerichtlichen Überprüfung hat es seither, soweit ersichtlich, nicht gegeben. Man hat sich - jedenfalls rechtlich - ein Stück weit damit abgefunden.

Der sog. Klima-Beschluss des BVerfG vom 24.3.2021 gibt allerdings Veranlassung, die Frage der VerfassungsmäBigkeit der 10-H-Regelung gänzlich neu zu stellen, weil diese Gerichtsentscheidung das verfassungsrechtliche $\mathrm{Ge}$ wicht des Klimaschutzes in mehrfacher Hinsicht deutlich gestärkt hat. Vor diesem Hintergrund werden im Folgenden zunächst die Entstehungsgeschichte der 10-H-Regelung und die aktuelle Rechtslage skizziert (2). AnschlieBend wird die rechtliche und tatsächliche Wirkung der 10-H-Regelung analysiert (3). Sodann wird die Frage der

Prof. Dr. Kurt Faßbender, Lehrstuhl für Öffentliches Recht, insbesondere Umwelt- und Planungsrecht,

Geschäftsführender Direktor des Instituts für Umweltund Planungsrecht, Universität Leipzig,

Leipzig, Deutschland
Verfassungsmäßigkeit der Regelung vor dem Hintergrund des Klima-Beschlusses des BVerfG vom 24.3.2021 erneut aufgerollt (4). Am Ende werden die wesentlichen Ergebnisse zusammengefasst (5).

\section{Entstehungsgeschichte der 10-H-Regelung und aktuelle Rechtslage}

\subsection{Der rechtliche Rahmen für den Ausbau der Windenergie}

Wie im Folgenden unter 4.2.1 und 4.2.3.1 noch näher darzulegen sein wird, ist der weitere massive Ausbau der Windenergie geboten, um das nunmehr verfassungsrechtlich gebotene Ziel der Klimaneutralität zu erreichen. Hierzu bedarf es in einem Rechtsstaat entsprechender rechtlicher Rahmenbedingungen. Entscheidende Bedeutung kommt dabei - neben der Förderung durch das EEG - dem Bauplanungsrecht zu. Denn jede Windenergieanlage muss auch bauplanungsrechtlich zulässig sein.

Die Errichtung von zur allgemeinen Energieversorgung bestimmten Windkraftanlagen kommt aufgrund deren Höhe nur außerhalb von bebauten Ortschaften im sog. AuBenbereich in Betracht, der nach der allgemeinen Konzeption des $₫ 35$ BauGB grundsätzlich von Bebauung freigehalten werden soll. Dennoch ist bei Windkraftanlagen im Gegensatz zu Solarfreiflächenanlagen zur Erreichung des Baurechts gerade kein Bebauungsplan erforderlich, weil erstere gemäß $\$ 35$ Abs. 1 Nr. 5 BauGB im Außenbereich privilegiert sind.

Die Gemeinden und die Träger der Raumordnungsplanung haben freilich gemäß $₫ 35$ Abs. 3 S. 3 BauGB die Möglichkeit, auf die Errichtung von Windenergieanlagen Einfluss zu nehmen, indem sie durch Darstellungen im Flächennutzungsplan oder als Ziele der Raumordnung eine Ausweisung an anderer Stelle vorsehen. Dies kann dazu führen, dass die Errichtung von Windenergieanlagen nur auf den ausgewiesenen Flächen zulässig ist. Eine solche Konzentrationsflächenplanung darf indessen nach der Rechtsprechung des BVerwG nicht auf eine ,verkappte Verhinderungsplanung“ hinauslaufen. Der Windenergie muss im Gegenteil entsprechend dem in $\$ 35$ Abs. 1 Nr. 5 BauGB zum Ausdruck kommenden gesetzgeberischen Willen durch ausreichend große privilegierte Flächen ,,in substanzieller Weise Raum verschafft" werden. ${ }^{1}$

Allerdings ist speziell mit Blick auf die hier interessierenden Abstände zur Wohnbebauung darauf hinzuweisen, dass vor allem das von der Rechtsprechung entwickelte Verbot einer „optisch bedrängenden Wirkung“ im Ergebnis dazu führt, dass Windkraftanlagen einen Abstand von min-

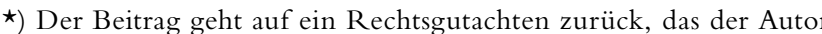
kürzlich im Auftrag der BayernSPD Landtagsfraktion erstellt hat.

1) Vgl. grundlegend BVerwG, Urt. v. 17.12.2002 - 4 C 15/01; BVerwG, Urt. v. 13.3.2003 - 4 C 4/02. 
destens der dreifachen Anlagenhöhe zur nächstgelegenen Wohnbebauung einhalten müssen (sog. 3-H-Regelung). ${ }^{2}$ Hinzu kommen immissionsschutzrechtliche Vorgaben zum Schutz vor Lärm, aus denen sich ebenfalls bestimmte Abstände zur Wohnbebauung ergeben. ${ }^{3}$

Demzufolge ist bereits an dieser Stelle festzuhalten, dass das geltende Recht und die Rechtsprechung bereits vor Einführung der 10-H-Regelung dem berechtigten Anliegen des Schutzes der Wohnbebauung vor einer, ,optisch bedrängenden Wirkung“" Rechnung getragen haben. Bemerkenswert ist weiterhin, dass bereits diese Abstandsvorgaben in der Fachliteratur teilweise als zu weitgehend empfunden werden, weil sie die Flächenverfügbarkeit deutlich beschränken und häufig das Repowering, also die Ersetzung vorhandener Windenergieanlagen durch neue leistungsfähigere Anlagen verhindern. ${ }^{4}$

\subsection{Die Einführung einer Öffnungsklausel im Bangesetzbuch}

Dessen ungeachtet hat der Bundesgesetzgeber im Jahr 2014 als Reaktion auf lokale Widerstände gegen Windenergieanlagen in $\$ 249$ Abs. 3 BauGB eine sog. „Öffnungsklausel“" eingeführt, die es den Ländern ermöglichte, die zuvor genannte Privilegierung der Windenergie im Außenbereich ( $\$ 35$ Abs. 1 Nr. 5 BauGB) bis zum 31.12.2015 unter die Bedingung zu stellen, dass die Anlage einen gewissen Abstand zu den im Landesrecht bestimmten Nutzungsarten einhält. Dabei sollten die Einzelheiten, insbesondere zur Abstandsfestlegung und zu den Auswirkungen der festgelegten Abstände auf Ausweisungen in geltenden Flächennutzungsplänen und Raumordnungsplänen, in den Landesgesetzen geregelt werden. Schließlich konnten die Länder in ihren Landesgesetzen auch Abweichungen von den festgelegten Abständen zulassen. ${ }^{5}$ Diese bundesrechtliche Regelung wurde bereits im Gesetzgebungsverfahren vielfach kritisch gesehen. ${ }^{6}$

\subsection{Einführung der 10-H-Regelung und Entscheidung des BayVerfGH}

Von der ursprünglichen Öffnungsklausel hat allein der Freistaat Bayern Gebrauch gemacht, indem Art. 82 BayBO im Jahr 2014 folgende Fassung erhielt:

„Art. 82 Windenergie und Nutzungsänderung ehemaliger
landwirtschaftlicher Gebäude
(1) $\$ 35$ Abs. 1 Nr. 5 BauGB findet auf Vorhaben, die der
Erforschung, Entwicklung oder Nutzung der Windenergie
dienen, nur Anwendung, wenn diese Vorhaben einen Min-
destabstand vom 10 -fachen ihrer Höhe zu Wohngebäuden in
Gebieten mit Bebauungsplänen (\$ 30 BauGB), innerhalb im
Zusammenhang bebauter Ortsteile ( $(\$ 4$ BauGB) - sofern
in diesen Gebieten Wohngebäude nicht nur ausnahmsweise
zulässig sind- und im Geltungsbereich von Satzungen nach
$\$ 35$ Abs. 6 BauGB einhalten. S 35 Abs. 6 BauGB einhalten.
(2) Höhe im Sinn des Abs. 1 ist Radius des Rotors. Der Abstand bemisst sich von der Mitte des Mastfußes bis zum nächstgelegenen Wohngebäude, das im jeweiligen Gebiet im Sinn des Abs. 1 zulässigerweise errichtet wurde bzw. errichtet werden kann.

(3) Soll auf einem gemeindefreien Gebiet ein Vorhaben nach Abs. 1 errichtet werden und würde der in Abs. 1 beschriebene Mindestabstand auch entsprechende Wohngebäude auf dem Gebiet einer Nachbargemeinde einschließen, gilt hinsichtlich dieser Gebäude der Schutz der Abs. 1 und 2, solange und soweit die Gemeinde nichts anderes in einem ortsüblich bekannt gemachten Beschluss feststellt.

(4) Abs. 1 und 2 finden keine Anwendung,

1. wenn in einem Flächennutzungsplan für Vorhaben der in Abs. 1 beschriebenen Art vor dem 21. November 2014 eine Darstellung für die Zwecke des $\int 35$ Abs. 3 Satz 3 BauGB erfolgt ist,
2. soweit und sobald die Gemeinde der Fortgeltung der Darstellung nicht bis einschließlich 21. Mai 2015 in einem ortsüblich bekannt gemachten Beschluss widerspricht und

3. soweit und sobald auch eine betroffene Nachbargemeinde der Fortgeltung der Darstellung nicht bis einschließlich 21. Mai 2015 in einem ortsüblich bekannt gemachten Beschluss widerspricht; als betroffen gilt dabei eine Nachbargemeinde, deren Wohngebäude in Gebieten im Sinn des Abs. 1 in einem geringeren Abstand als dem 10-fachen der Höhe der Windkraftanlagen, sofern der Flächennutzungsplan jedoch keine Regelung enthält, maximal in einem Abstand von $2000 \mathrm{~m}$, stehen (5) Bei der Aufstellung von Bauleitplänen, die für Vorhaben nach Abs. 1 einen geringeren als den dort beschriebenen Mindestabstand festsetzen wollen, ist im Rahmen der Abwägung nach $\int 1$ Abs. 7 BauGB auf eine einvernehmliche Festlegung mit betroffenen Nachbargemeinden hinzuwirken. Abs. 4 Nr. 3 Halbsatz 2 gilt entsprechend.

(6) Die Frist nach $\int 35$ Abs. 4 Satz 1 Nr. 1 Buchst. c BauGB ist nicht anzuwenden."

Auch diese landesrechtliche Regelung wurde vielfach, nicht zuletzt aus verfassungsrechtlichen Gründen, kritisch gesehen. $^{7}$

Mit einem Teil der verfassungsrechtlichen Einwände musste sich der BayVerfGH aus der Perspektive der Bayerischen Verfassung aus Anlass einer sog. Popularklage und zweier sog. Meinungsverschiedenheiten auseinandersetzen, von denen eine von der SPD-Fraktion im Bayerischen Landtag eingeleitet wurde. Wie bereits erwähnt, hat der BayVerfGH die 10-H-Regelung in seiner Entscheidung vom 9.5.2016 im Wesentlichen gebilligt. Als verfassungswidrig wurde allein die in Art. 82 Abs. 5 BayBO den Gemeinden auferlegte Pflicht angesehen, bei der Aufstellung von Bauleitplänen, die für Vorhaben der Windenergienutzung einen geringeren als den Mindestabstand festsetzen wollen, im Rahmen der Abwägung nach $\$ 1$ Abs. 7 BauGB auf eine einvernehmliche Festlegung mit betroffenen Nachbargemeinden hinzuwirken. Diese Regelung stand nach Ansicht des BayVerfGH in einem ,offensichtlichen und schwerwiegenden Widerspruch zur Kompetenzordnung des Grundgesetzes“ und verstieß deshalb gegen das Rechtsstaatsprinzip der Bayerischen Verfassung. ${ }^{8}$ Auf die übrigen Inhalte der Entscheidung wird im Folgenden unter 4. noch näher eingegangen.

\subsection{Die jüngste Änderung der Öffnungsklausel im Bangesetzbuch}

Im August 2020 hat der Bundesgesetzgeber nach zähen Auseinandersetzungen die Öffnungsklausel in $\$ 249$ Abs. 3 BauGB geändert. ${ }^{9}$ Hierdurch ist zunächst einmal die zeitliche Befristung entfallen; die Norm gilt nunmehr zeitlich unbeschränkt. Abs. 3 S. 1 neuer Fassung (n. F.) stellt zudem auf bestimmte Mindestabstände ab, für die in Satz 2 n. F. eine neue Höchstgrenze von $1000 \mathrm{~m}$ normiert wird. Im Übrigen können die Länder weiterhin die Einzelheiten, insbe-

2) Vgl. OVG Münster, Urt. v. 9.8.2006 - 8 A 3726/05; bestätigt durch BVerwG, Beschl. v. 11.12.2006 - 4 B 72/06.

3) Vgl. etwa Albrecht/Zschiegner, NVwZ 2015, 1093, 1095.

4) Vgl. etwa Agatz, ZUR 2020, 584, $589 \mathrm{f}$.

5) Vgl. das Gesetz zur Einführung einer Länderöffnungsklausel zur Vorgabe von Mindestabständen zwischen Windenergieanlagen und zulässigen Nutzungen v. 15.7.2014, BGBl. 2014 I, S. $954 \mathrm{f}$.

6) Vgl. näher dazu mit weiteren Nachweisen Krautzberger/Stüer, BauR 2014, 1403, $1407 \mathrm{f}$.

7) Vgl. etwa die verfassungsrechtlichen Einwände bei Albrecht/ Zschiegner, NVwZ 2015, 1093 ff.; Helmes/Klöcker, REE 2015, 81 ff.; Würfel/Werner, BayVBl. 2015, $109 \mathrm{ff}$.

8) Vgl. BayVerfGH, Entsch. v. 9.5.2016 - Vf. 14-VII-14, Vf. 3-VIII15, Vf. 4-VIII-15.

9) Siehe das BGBl. 2020 I, S. 1728 ff.; vgl. näher zur Entstehungsgeschichte Decker, ZfBR 2021, 19. 
sondere zur Abstandsfestlegung und zu den Auswirkungen der festgelegten Abstände auf Ausweisungen in geltenden Flächennutzungsplänen und Raumordnungsplänen, in ihren Landesgesetzen regeln. Schließlich wurde in $\$ 249$ BauGB ein neuer Abs. 3 S. 4 eingefügt, der im Schrifttum bereits als „Bayern-Klausel“ bezeichnet wurde, weil die Vorschrift der auf $\$ 249$ Abs. 3 BauGB a.F. beruhenden 10-H-Regelung eine Art „Bestandsschutz“ vermittelt. ${ }^{10}$ Die zuvor genannte neue Öffnungsklausel gibt den Ländern nämlich, wie erwähnt, nur die Möglichkeit, einen Mindestabstand von max. $1000 \mathrm{~m}$ festzulegen, während die 10-H-Regelung in der Sache dazu führt, dass der Abstand von Windenergieanlagen zur Wohnbebauung teilweise $2000 \mathrm{~m}$ und mehr betragen muss. Die 10-H-Regelung wäre also ohne die „Bayern-Klausel“ schon aus Kompetenzgründen nicht mehr zulässig.

\section{Die kontraproduktiven Wirkungen der 10-H-Regelung}

Damit sind bereits die Wirkungen der 10-H-Regelung angesprochen, die es näher zu beleuchten gilt, um ihre Tragweite für den Klimaschutz sachgerecht erfassen zu können.

\subsection{Die rechtliche Wirkung der 10-H-Regelung}

Rein rechtlich betrachtet bewirkt die 10-H-Regelung zunächst einmal „,lediglich“ eine „Entprivilegierung“ der den Abstand nicht einhaltenden Windenergieanlagen im bauplanungsrechtlichen Außenbereich. Angesichts dessen weist der BayVerfGH in seiner zuvor genannten Entscheidung ausdrücklich darauf hin, dass entsprechende Windenergieanlagen damit nicht zwangsläufig unzulässig werden, sondern „nur“ deutlich strengeren Genehmigungsvoraussetzungen unterliegen. ${ }^{11}$ Damit stellt der BayVerfGH auf den Umstand $\mathrm{ab}$, dass Vorhaben, die nicht (mehr) nach $\$ 35$ Abs. 1 BauGB privilegiert sind, gemäß $₫ 35$ Abs. 2 BauGB als sonstige Vorhaben im Einzelfall zugelassen werden können. Eine entsprechende Genehmigung kommt aber tatsächlich kaum in Betracht, weil Windenergieanlagen in der Regel öffentliche Belange im Sinne des $₫ 35$ Abs. 3 S. 1 BauGB beeinträchtigen. ${ }^{12}$ Daher ist selbst die Bayerische Staatsregierung in der Begründung zu ihrem Gesetzentwurf zur 10-H-Regelung davon ausgegangen, dass die Errichtung von entprivilegierten Windkraftanlagen nach Inkrafttreten der 10-H-Regelung regelmäßig nur noch im Wege einer Bauleitplanung erfolgen kann. ${ }^{13}$

Das führt zu einem weiteren Argument, das gegen die verbreitete Einschätzung angeführt wird, die 10-H-Regelung führe zu einer übermäßigen Beschränkung des Ausbaus der Windenergie im Freistaat Bayern: die fortbestehende Möglichkeit der Gemeinden, durch Bebauungspläne geringere Abstände zuzulassen. Die Bayerische Staatsregierung macht nämlich in der Begründung zu ihrem Gesetzentwurf von 2014 explizit geltend, nach dem Gesamtkonzept des Gesetzes werde der Windenergie gerade durch die Möglichkeit der Gemeinden, durch Bebauungspläne geringere Abstände zuzulassen, weiterer Raum verschafft. ${ }^{14}$

Dieses Argument wird teilweise auch im Schrifttum ohne nähere Prüfung gegen den Einwand ins Feld geführt, die 10-H-Regelung wirke wie eine "Windkraftbremse“. ${ }^{15}$ Hierzu hat allerdings selbst der BayVerfGH zutreffend bemerkt, der bundesrechtliche Privilegierungstatbestand des $\$ 35$ Abs. 1 Nr. 5 BauGB erfordere, ,notwendigerweise das Vorhandensein von Flächen im Außenbereich, die außerhalb des landesrechtlich vorgeschriebenen Mindestabstands liegen. Diesem Erfordernis kann nicht durch die Aussicht auf eine - im Umfang zudem ungewisse - gemeindliche Bauleitplanung Rechnung getragen werden." 16

Somit ist als Zwischenergebnis festzuhalten, dass die 10-HRegelung zwar rechtlich „nur“ eine „Entprivilegierung“ der den Abstand nicht einhaltenden Windenergieanlagen im bauplanungsrechtlichen Außenbereich bewirkt. Dies führt aber faktisch dazu, dass die Realisierung solcher Anlagen weitestgehend verhindert wird, weil eine Zulassung nach \$35 Abs. 2 BauGB in der Regel nicht in Betracht kommt und die Aussicht auf eine im Umfang gemeindliche Bauleitplanung keine auch nur halbwegs adäquate Alternative darstellt.

\subsection{Die tatsächliche Wirkung der 10-H-Regelung}

Dieser Befund deckt sich mit den empirischen Erkenntnissen zur tatsächlichen Wirkung der 10-H-Regelung. Hierbei ist zunächst einmal zu berücksichtigen, dass bei modernen Windenergieanlagen nach der Erkenntnis des Umweltbundesamtes (UBA) deshalb Gesamthöhen von $200 \mathrm{~m}$ und mehr zugrunde zu legen sind, weil sie im windschwächeren Binnenland errichtet werden. Deshalb geht das $U B A \mathrm{zu}$ Recht davon aus, dass aus der 10-H-Regel bei neuen Windenergieanlagen faktisch ein Siedlungsabstand von $2000 \mathrm{~m}$ resultiert. ${ }^{17}$

Daher greift der Hinweis des BayVerfGH zu kurz, dass Windkraftanlagen niedrigerer Höhe nicht außer Betracht bleiben könnten, auch wenn sie, zumal mit Blick auf die Absenkung der finanziellen Förderung durch das EEG, weniger rentabel sind. ${ }^{18}$ Denn es geht hier nicht nur um die wirtschaftliche Rentabilität, die zunächst einmal bloß den Anlagenbetreibern zugutekommt, sondern darum, dass der Ausbau der Windkraft effektiv vonstattengeht, damit die Energiewende gelingt, die wiederum nach dem Klima-Beschluss des BVerfG, wie im Folgenden unter 4 zu zeigen sein wird, letztlich verfassungsrechtlich geboten ist. Im Ubrigen ist die wirtschaftliche Rentabilität eine wichtige Voraussetzung dafür, dass Personen und Unternehmen weiterhin in den Ausbau der Windenergie investieren. Hierfür bedarf es wiederum entsprechender Flächen, auf denen Windenergieanlagen in zulässiger Weise errichtet werden können. Diese sog. Potenzialflächen werden jedoch durch die 10-HRegel ausgerechnet im Bundesland mit der größten Landesfläche in unverhältnismäßiger Weise verkleinert.

Dabei ist zu berücksichtigen, dass sich die Landesflächen, die nach Abzug der Flächen, die durch die 10-H-Regel wegfallen, als sog. Bruttoflächen verbleiben, weiter erheblich reduzieren, wenn von ihnen diejenigen Bereiche abgezogen werden, in denen Windenergieanlagen trotz ihrer Privilegierung rechtlich - aus anderen Gründen als dem fehlenden Abstand - nicht zugelassen oder aus tatsächlichen Gründen nicht sinnvoll betrieben werden können - was auch der BayVerfGH in seiner Entscheidung vom 9. 5.2016 ausdrücklich anerkennt. ${ }^{19}$ Dies liegt zum einen an dem zu-

10) Siehe Decker, ZfBR 2021, 19, 20.

11) Vgl. BayVerfGH, Entsch. v. 9.5.2016-Vf. 14-VII-14, Vf. 3-VIII15, Vf. 4-VIII-15, Rdnr. 120

12) So zu Recht Würfel/Werner, BayVBl. 2015, 109, 111.

13) Vgl. Bayerischer Landtag, Drs. 17/2137, S. 7.

14) Vgl. Bayerischer Landtag, Drs. 17/2137, S. 7.

15) So etwa Ludwigs, NVwZ 2015, 986, 990.

16) BayVerfGH, Entsch. v. 9.5.2016 - Vf. 14-VII-14, Vf. 3-VIII-15, Vf. 4-VIII-15, Rdnr. 134; zustimmend und mit gleicher Stoßrichtung Winkler/Stückemann, UPR 2016, 516, 519: Der BayVerfGH habe ,richtigerweise die (nur theoretische) Möglichkeit, planungsrechtlich Flächen für Windkraftanlagen auch innerhalb der bestehenden Abstandsflächen auszuweisen, nicht berücksichtigt.“

17) Vgl. $U B A$, Auswirkungen von Mindestabständen zwischen Windenergieanlagen und Siedlungen - Auswertung im Rahmen der UBA-Studie „,Flächenanalyse Windenergie an Land“, Positionspapier vom März 2019, S. 9, Stand 5.10.2021, abrufbar unter https://www.umweltbundesamt.de/sites/default/files/medien/ 1410/publikationen/2019-03-20_pp_mindestabstaende-windenergieanlagen.pdf.

18) Vgl. BayVerfGH, Entsch. v. 9. 5.2016 - Vf. 14-VII-14, Vf. 3-VIII15, Vf. 4-VIII-15, Rdnr. 135.

19) Vgl. BayVerfGH, Entsch. v. 9.5.2016-Vf. 14-VII-14, Vf. 3-VIII15, Vf. 4-VIII-15, Rdnr. 137. 
vor angedeuteten Umstand, dass Windenergieanlagen naturgemäß auf ein bestimmtes Windaufkommen angewiesen sind und ausreichend windhöffige Gebiete vor allem in den Bundesländern, die nicht in Küstennähe liegen, aufgrund ihrer Topographie ohnehin ein knappes Gut sind. Zum anderen werden die verbleibenden Flächen häufig durch rechtliche Gründe wie nationale und EU-rechtlich vorgegebene Vorgaben des Naturschutzrechts, insbesondere des Artenschutzrechts, sowie durch vorgegebene Abstände zu Luftverkehrseinrichtungen weiter erheblich eingeschränkt. ${ }^{20}$

Vor diesem Hintergrund haben die Antragsteller vor dem BayVerfGH unwidersprochen vorgetragen, dass die verbleibende privilegierte Fläche nach Ermittlungen durch die Oberste Baubehörde im zuständigen Bayerischen Staatsministerium unter Berücksichtigung sonstiger Ausschlusskriterien nur $0,01 \%$ der Landesfläche umfasse. ${ }^{21}$ Diese Annahme wird durch das zuvor erwähnte Positionspapier des $U B A$ eindrucksvoll unterstrichen. Denn in diesem Positionspapier hat das $U B A$ die Ergebnisse eines seinerzeit laufenden Forschungsvorhabens zu den Auswirkungen pauschaler Siedlungsabstände auf die Flächenkulisse für die Windenergienutzung ausgewertet. Danach reduziert bereits ein pauschaler Siedlungsabstand von $1000 \mathrm{~m}$ die deutschlandweite Flächenkulisse um 20 bis $50 \%$. Bei einem Abstand von $1500 \mathrm{~m}$ verbleiben nur noch 15 bis $40 \%$ der Fläche. Und die 10-H-Regelung führt nach dem vom UBA in Auftrag gegebenen Forschungsvorhaben zu einer Reduzierung der Fläche für die Windenergienutzung um 85 bis $97 \% .^{22}$

Diese kontraproduktive Wirkung der 10-H-Regelung auf den Ausbau der Windenergie lässt sich auch anhand der Entwicklung der Genehmigungen neuer Windenergieanlagen in Bayern im Zeitraum 2010-2018 belegen. Hierzu heißt es im Positionspapier des $U B A$ auf der Basis von Zahlen des Bayerischen Staatsministeriums für Wirtschaft und Medien, Energie und Technologie sowie der Bundesnetzagentur:

\section{„Während die neu genehmigte Windenergieleistung im Zeit- raum 2010-2014, getrieben durch die Entwicklung ertrags- starker Binnenlandanlagen, von $143 \mathrm{MW}$ im Jahr 2010 auf 637 MW im Jahr 2014 zunahm, brach sie in den Folgejah- ren stark ein .... Während im Jahr 2016 bundesweit Geneh- migungen über insgesamt $9400 \mathrm{MW}$ ausgestellt wurden - ein Rekordwert, der maßgeblich auf Vorzieheffekte der EEG-No- velle 2017 zurückzuführen ist - wurden in Bayern lediglich 210 MW neu genehmigt. Für das Jahr 2018 sind nur noch Genehmigungen über insgesamt $50 \mathrm{MW}$ in Bayern verzeich- net, dies entspricht lediglich $3 \%$ der im gleichen Zeitraum deutschlandweit ausgestellten Genehmigungen. Der Wind- energieausbau in Bayern wird in den kommenden Jahren so- mit nahezu vollkommen zum Erliegen kommen. ${ }^{23}$}

Schließlich wirkt sich die 10-H-Regelung überaus negativ auf die Möglichkeiten eines Repowering, also die Ersetzung vorhandener Windenergieanlagen durch neue leistungsfähigere Anlagen aus. Dies ist gerade in der jetzigen Situation besonders misslich, weil seit dem 1.1.2021 erstmals Windenergieanlagen das Ende des 20-jährigen Förderzeitraums nach EEG erreichen. Dabei ist nach dem Positionspapier des UBA davon auszugehen, dass etwa $53 \%$ der bestehenden Windenergieanlagen aus planungsrechtlicher Sicht repoweringfähig wären. Dieses Repoweringpotential reduziert sich jedoch bei Abständen von 1500 bis $2000 \mathrm{~m}$ auf 16 bis $1 \%$ der heutigen Anlagenstandorte. ${ }^{24}$

Somit führt die 10-H-Regelung dazu, dass auch die Ersetzung vorhandener Windenergieanlagen in hohem Maße torpediert wird, obwohl empirische Studien zeigen, dass die Zustimmung zur Windenergienutzung im eigenen räumlichen Umfeld steigt, wenn die Anwohner`innen bereits Erfahrung mit Windenergieanlagen gemacht haben. ${ }^{25}$

\section{Neubewertung der Verfassungsmäßigkeit der 10-H-Regelung}

Angesichts derart negativer Effekte auf den Bau neuer Windenergieanlagen und die Ersetzung vorhandener Windenergieanlagen wurde im rechtswissenschaftlichen Schrifttum bereits im Jahre 2016 darauf hingewiesen, dass die 10-HRegel zu „Friktionen mit Art. 20a GG“ führe. ${ }^{26}$ Dieser Einschätzung kommt nach dem Klima-Beschluss des BVerfG vom 24.3.2021 ein deutlich größeres Gewicht zu.

\subsection{Die Notwendigkeit einer Neubewertung} nach dem Klima-Beschluss des BVerfG

Denn das BVerfG hat in seinem Klima-Beschluss, der zu Recht als historisch oder gar epochal eingestuft wird, seine teils sehr konkreten Forderungen zur Begrenzung der $\mathrm{CO}_{2}$-Emissionen mit dem Ziel der Herstellung von Klimaneutralität ganz maßgeblich aus Art. 20a GG hergeleitet, der den Staat verpflichtet, die natürlichen Lebensgrundlagen auch in Verantwortung für die künftigen Generationen zu schützen. ${ }^{27}$ Das ist eine der zentralen verfassungsrechtlichen Neuerungen dieser Entscheidung, weil Art. 20a GG bislang - auch vom BVerfG - fast ausschließlich als Staatszielbestimmung verstanden wurde und bislang rechtlich eher ein Schattendasein fristete. ${ }^{28}$ Angesichts dessen heißt es im rechtswissenschaftlichen Schrifttum beispielsweise, das BVerfG habe Art. 20a GG aus seinem „,verfassungsgerichtlichen ,Dornröschenschlaf“" erweckt. ${ }^{29}$

Daneben enthält der Klima-Beschluss, wie im Folgenden zu zeigen sein wird, weitere zentrale Neuansätze,

20) Vgl. UBA, Analyse der kurz- und mittelfristigen Verfügbarkeit von Flächen für die Windenergienutzung an Land, Abschlussbericht von Marian Bons u.a., Juni 2019, S. 20, 87 und 148 , Stand 5.10.2021, abrufbar unter https://www.umweltbundesamt.de/sites/default/files/medien/376/publikationen/climate_ change_38_2019_flaechenanalyse_windenergie_an_land.pdf.

21) Vgl. BayVerfGH, Entsch. v. 9. 5.2016-Vf. 14-VII-14, Vf. 3-VIII15, Vf. 4-VIII-15, Rdnr. 137.

22) Vgl. Vgl. $U B A$, Auswirkungen von Mindestabständen zwischen Windenergieanlagen und Siedlungen - Auswertung im Rahmen der UBA-Studie „Flächenanalyse Windenergie an Land“, Positionspapier vom März 2019, Stand 5.10.2021, abrufbar unter https://www.umweltbundesamt.de/sites/default/files/medien/ 1410/publikationen/2019-03-20_pp_mindestabstaende-windenergieanlagen.pdf, S. $14 \mathrm{f}$.

23) Vgl. Vgl. UBA, Auswirkungen von Mindestabständen zwischen Windenergieanlagen und Siedlungen - Auswertung im Rahmen der UBA-Studie „Flächenanalyse Windenergie an Land“, Positionspapier vom März 2019, Stand 5.10.2021, abrufbar unter https://www.umweltbundesamt.de/sites/default/files/medien/ 1410/publikationen/2019-03-20_pp_mindestabstaende-windenergieanlagen.pdf, S. 10 (Hervorhebung nur hier).

24) Vgl. Vgl. UBA, Auswirkungen von Mindestabständen zwischen Windenergieanlagen und Siedlungen - Auswertung im Rahmen der UBA-Studie „Flächenanalyse Windenergie an Land“, Positionspapier vom März 2019, Stand 5.10.2021, abrufbar unter https://www.umweltbundesamt.de/sites/default/files/medien/ 1410/publikationen/2019-03-20_pp_mindestabstaende-windenergieanlagen.pdf, S. 18.

25) Vgl. Vgl. UBA, Auswirkungen von Mindestabständen zwischen Windenergieanlagen und Siedlungen - Auswertung im Rahmen der UBA-Studie „Flächenanalyse Windenergie an Land“, Positionspapier vom März 2019, Stand 5.10.2021, abrufbar unter https://www.umweltbundesamt.de/sites/default/files/medien/ 1410/publikationen/2019-03-20_pp_mindestabstaende-windenergieanlagen.pdf, S. 19.

26) Siehe Winkler/Stückemann, UPR 2016, 516, 519

27) Vgl. BVerfG, Beschl. v. 24.3.2021 - 1 BvR 2656/18, 1 BvR 78/29, 1 BvR 96/20, 1 BvR 288/20, Leitsatz 2, Rdnr. 188 ff., 193, $243 \mathrm{ff}$.

28) Vgl. etwa die Analyse und Nachweise bei Berkemann, DÖV 2021, $701,704 \mathrm{ff}$

29) So etwa Calliess, ZUR 2021, 355, 357. 
die verfassungsrechtlich den „Weg zu neuen Ufern“ weisen. ${ }^{30}$ Diese Neuerungen haben in der rechtswissenschaftlichen Literatur sogar zu der These geführt, dass zentrale Regelungen des deutschen und europäischen Umweltrechts schlicht verfassungswidrig seien. ${ }^{31}$ Das kann hier aus Raumgründen nicht näher aufbereitet werden. Es zeigt aber, welche weitreichenden Konsequenzen aus dem Beschluss des BVerfG mit Blick auf solche Vorschriften gezogen werden, die unter Klimaschutzgesichtspunkten besonders relevant sind.

\subsection{Vereinbarkeit der 10-H-Regelung mit dem neuen Grundrecht auf intertemporale Freiheitssicherung}

\subsubsection{Die zugrundeliegenden Annahmen des BVerfG}

Eine der weiteren zentralen Neuerungen des Klima-Beschlusses ist die Aussage des BVerfG, dass das Grundgesetz unter bestimmten Voraussetzungen zur Sicherung grundrechtsgeschützter Freiheit über die Zeit und zur verhältnismäßigen Verteilung von Freiheitschancen über die Generationen verpflichte. Das sei nicht nur durch Art. 20a GG objektivrechtlich geboten. Vielmehr schützten die Grundrechte die Beschwerdeführenden subjektivrechtlich als „,intertemporale Freiheitssicherung“ vor einer einseitigen Verlagerung der durch Art. 20a GG aufgegebenen Treibhausgasminderungslast in die Zukunft. ${ }^{32}$

Dieses neue Grundrecht auf intertemporale Freiheitssicherung sah das BVerfG im konkreten Fall als verletzt an, weil der Gesetzgeber im streitgegenständlichen Bundes-Klimaschutzgesetz (KSG) nach Ansicht des Gerichts keine ausreichenden Vorkehrungen getroffen habe, die - wegen der gesetzlich bis 2030 zugelassenen Emissionen in späteren Zeiträumen möglicherweise sehr hohen - Emissionsminderungspflichten grundrechtsschonend zu bewältigen. ${ }^{33}$ Dahinter steht die tatsächliche Überlegung, dass die Auswirkungen von $\mathrm{CO}_{2}$-Emissionen auf die Erdtemperatur weitgehend unumkehrbar sind und dass global ein bestimmtes $\mathrm{CO}_{2}$-Budget eingehalten werden muss, wenn das in $₫ 1$ S. 3 KSG in Anlehnung an das Pariser Klimaschutzabkommen normierte und verfassungsrechtlich relevante Ziel, den Anstieg der globalen Durchschnittstemperatur auf deutlich unter $2{ }^{\circ} \mathrm{C}$ und möglichst auf $1,5^{\circ} \mathrm{C}$ gegenüber dem vorindustriellen Niveau zu begrenzen, eingehalten werden soll. Aus diesem globalen Restbudget könne ein nationales $\mathrm{CO}_{2}$-Budget abgeleitet werden, das für Deutschland nach Berechnungen des SRU ab 2020 insgesamt 6,7 Gigatonnen betrage und das bis 2030 weitgehend aufgezehrt werde, weil für die Zeit danach nur weniger als $1 \mathrm{Gi}-$ gatonne bliebe. ${ }^{34}$

Der Klima-Beschluss vermittelt also, vereinfacht gesprochen, ein durch die Grundrechte vermitteltes Recht auf Abwehr von systematischen Verlagerungen von $\mathrm{CO}_{2}$-Emissionen in die Zeit nach 2030. Dabei ist wiederum das vom BVerfG sog. Klimaschutzgebot des Art. 20a GG zu beachten, welches die Gesetzgebung - nach Ansicht des Gerichts verfassungsrechtlich maßgeblich - durch das Ziel konkretisiert hat, die Erwärmung der Erde bis 2050 auf deutlich unter $2^{\circ} \mathrm{C}$ und möglichst auf $1,5^{\circ} \mathrm{C}$ gegenüber dem vorindustriellen Niveau zu begrenzen. Insofern muss die „,dem Staat" aufgegebene Pflicht zum Klimaschutz auch auf die Herstellung von Klimaneutralität abzielen..$^{35}$

Schließlich weist das BVerfG ausdrücklich darauf hin, dass die Vereinbarkeit mit Art. 20a GG Voraussetzung für die verfassungsrechtliche Rechtfertigung staatlicher Eingriffe in Grundrechte ist und dass das relative Gewicht des Klimaschutzgebots in der Abwägung bei fortschreitendem Klimawandel weiter zunimmt. ${ }^{36}$

\subsubsection{Die dahinter stehende Frage der Verteilungs- und Umweltgerechtigkeit}

Hinter diesen Ausführungen steht unter anderem die Annahme, dass Freiheit auch ein verteilungsfähiges (gesell- schaftliches) Gut ist, was sodann die grundlegende Frage nach der Verteilungsgerechtigkeit nach sich zieht. Dies ist wiederum in der Tat eine Frage, die allgemein unter dem Gesichtspunkt der Umweltgerechtigkeit diskutiert wird. ${ }^{37}$

\subsubsection{Konsequenzen für die 10-H-Regelung}

Möchte man die Frage nach den Konsequenzen für die verfassungsrechtliche Bewertung der 10-H-Regelung sachgerecht beantworten, so muss man zunächst einmal die klimapolitischen und energiewirtschaftlichen Rahmenbedingungen in den Blick nehmen (4.2.3.1). Denn erst aus diesen Rahmenbedingungen erhellt sich, warum man sich auf den Standpunkt stellen kann, dass die 10-H-Regelung das neue Grundrecht auf intertemporale Freiheitssicherung in relevanter Weise gefährdet (4.2.3.2). Dabei ist auch die im letzten Abschnitt angesprochene Frage der Verteilungs- und Umweltgerechtigkeit zu berücksichtigen (4.2.3.3). Es verbleibt hier freilich ein gewisser Klärungsbedarf (4.2.3.4).

\subsubsection{Klimapolitische und wirtschaftliche Rahmenbedingungen}

Zunächst einmal ist in tatsächlicher Hinsicht davon auszugehen, dass die Energiewirtschaft mit ca. 30 \% für den größten Anteil der $\mathrm{CO}_{2}$-Emissionen in Deutschland verantwortlich ist. Die Emissionen aus der Energiewirtschaft entstehen vor allem bei der Verbrennung fossiler Energieträger in Kraftwerken der öffentlichen Versorgung zur Bereitstellung von Strom und Wärme. Das zentrale Handlungsfeld der Energiewirtschaft und damit auch der Klimapolitik ist daher ein zielstrebiger und deutlicher Ausbau der erneuerbaren Energien. ${ }^{38}$

Diese sog. Energiewende schlägt sich auch im ErneuerbareEnergien-Gesetz (EEG) nieder, das als geltendes Bundesrecht - wie im Folgenden näher ausgeführt wird - auch bei der verfassungsrechtlichen Bewertung der 10-H-Regelung zu beachten ist. So bestand das Ziel des Gesetzes gemäß $\int 1$ Abs. 2 EEG bis Mitte 2020 darin, den Anteil des aus erneuerbaren Energien erzeugten Stroms am Bruttostromverbrauch bis zum Jahr 2025 auf 40 bis $45 \%$, bis zum Jahr 2035 auf 55 bis $60 \%$ und bis zum Jahr 2050 auf mindestens $80 \%$ zu steigern. Dieses Ziel wurde durch Gesetz vom 8. 8.2020 auf $65 \%$ bis zum Jahr 2030 angehoben (siehe $\$ 1$ Abs. 2 EEG n. F.). Schließlich wurde durch Gesetz vom 21.12.2020 in $\$ 1$ Abs. 3 EEG das Ziel festgeschrieben, dass vor dem Jahr 2050 der gesamte Strom, der im Staatsgebiet der Bundesrepublik Deutschland einschließlich der deutschen ausschließlichen Wirtschaftszone (Bundesgebiet) erzeugt oder verbraucht wird, treibhausgasneutral erzeugt wird. Dieses Fernziel ist, wie bereits erwähnt, aufgrund des

30) Siehe Berkemann, DÖV 2021, 701

31) Siehe Sinner, UPR 2021, $281 \mathrm{ff}$.

32) Vgl. BVerfG, Beschl. v. 24.3.2021 - 1 BvR 2656/18, 1 BvR 78/29, 1 BvR 96/20, 1 BvR 288/20, Leitsatz 4 sowie Rdnr. 166 , 183; vgl. hierzu und zum Folgenden auch Faßbender, NJW 2021, 2085, $2086 \mathrm{ff}$.

33) Siehe BVerfG, Beschl. v. 24.3.2021 - 1 BvR 2656/18, 1 BvR 78/29, 1 BvR 96/20, 1 BvR 288/20, Rdnr. 182.

34) Vgl. BVerfG, Beschl. v. 24.3.2021-1 BvR 2656/18, 1 BvR 78/29, 1 BvR 96/20, 1 BvR 288/20, Leitsatz 4 sowie Rdnr. 118 ff., 185, $208 \mathrm{ff}$. und 233.

35) Vgl. BVerfG, Beschl. v. 24.3.2021 - 1 BvR 2656/18, 1 BvR 78/29, 1 BvR 96/20, 1 BvR 288/20, Leitsatz 2 sowie Rdnr. 118 ff., 198 und $208 \mathrm{ff}$.

36) Vgl. BVerfG, Beschl. v. 24.3.2021 - 1 BvR 2656/18, 1 BvR 78/29, 1 BvR 96/20, 1 BvR 288/20, Leitsätze 2 a., 3 sowie Rdnr. 120, 185, 190, 198.

37) Siehe die treffende Analyse von Berkemann, DÖV 2021, 701, $712 \mathrm{f}$.

38) Vgl. zum Ganzen Bundesministerium für Umwelt, Naturschutz und nukleare Sicherheit $(B M U)$, Klimaschutz in Zahlen - Fakten, Trends und Impulse deutscher Klimapolitik, Ausgabe 2021, S. 29,31 . 
Klima-Beschlusses des BVerfG nunmehr verfassungsrechtlich durch Art. 20a GG abgesichert. ${ }^{39}$

Bei alledem kommt dem Ausbau der Windenergie an Land unter Klimaschutzgesichtspunkten eine besondere Rolle zu. Denn sie ist ein fundamentaler Pfeiler der Energiewende. Dies erhellt schon die Tatsache, dass die Windenergie an Land im Jahr 2020 erstmals den größten Beitrag aller Technologien zur Stromerzeugung lieferte und damit die Braunkohle ablöste. ${ }^{40}$ Diese besondere Rolle soll und muss die Windenergie an Land auch in Zukunft spielen. So geht der Bundesgesetzgeber in $\$ 4$ EEG 2021 davon aus, dass eine Steigerung der installierten Leistung von Windenergieanlagen an Land von derzeit rund 55 Gigawatt auf 71 Gigawatt im Jahr 2030 erforderlich ist, um das in $\$ 1$ Abs. 2 EEG 2021 normierte Ziel zu erreichen, den Anteil des aus erneuerbaren Energien erzeugten Stroms am Bruttostromverbrauch bis zum Jahr 2030 auf $65 \%$ zu steigern.

Es ist jedoch schon jetzt davon auszugehen, dass auf diesem Wege allein das vom BVerfG vorgegebene Ziel der Klimaneutralität nicht erreicht werden kann. Hierfür müssen nämlich neben der Energiewirtschaft zumindest zwei weitere Wirtschaftssektoren mit in den Blick genommen werden, die ebenfalls ganz wesentlich zu den klimapolitisch problematischen $\mathrm{CO}_{2}$-Emissionen beitragen.

Dies ist zum einen die Industrie, die nach der Energiewirtschaft der zweitgrößte Verursacher von $\mathrm{CO}_{2}$-Emissionen ist. Die $\mathrm{CO}_{2}$-Emissionen des Industriesektors entstehen vor allem in den energieintensiven Branchen Stahl, Chemie, Nichteisenmetalle, Zement, Kalk, Glas und Papier sowie bei der industriellen Eigenstromversorgung. Dabei entstehen rund zwei Drittel der Emissionen durch direkte Energiebereitstellung in der Industrie. Daher ist der beschleunigte Ausbau der erneuerbaren Stromerzeugung auch für den Industriesektor eine ,zentrale Grundvoraussetzung" für die Erreichung der Treibhausgasneutralität. Nur so kann der steigende Strombedarf des Sektors, der etwa durch die Elektrifizierung von Produktionsprozessen und die Herstellung von klimaneutralem Wasserstoff entsteht, gedeckt werden. ${ }^{41}$

Zum anderen ist der beschleunigte Ausbau der erneuerbaren Stromerzeugung auch für den Verkehrssektor als den drittgrößten Verursacher von $\mathrm{CO}_{2}$-Emissionen eine unerlässliche Voraussetzung für die Erreichung der Treibhausgasneutralität, weil hier die Umstellung auf elektrische Antriebe eine entscheidende Rolle spielt. ${ }^{42}$

Vor diesem Hintergrund ist mit $\operatorname{dem} U B A$ festzuhalten, dass die Bedeutung der Windenergie und gleichermaßen der Bedarf an geeigneten Flächen für die Errichtung von Windenergieanlagen weiter erheblich zunehmen werden. ${ }^{43}$ Denn der beschleunigte Ausbau der erneuerbaren Stromerzeugung ist nicht nur für die Energiewende unerlässlich, sondern eine Grundvoraussetzung für die Erreichung der Treibhausgasneutralität in den Bereichen Energie und Verkehr. Insofern hängt der Erfolg der Klimaschutzpolitik insgesamt auch vom Ausbau der Windenergie ab.

\subsubsection{Die 10-H-Regelung als mögliche Gefährdung} des Grundrechts auf intertemporale Freiheitssicherung

Wenn man nun aber bedenkt, dass dieser für den Klimaschutz essentielle Ausbau der Windenergie an Land durch die 10-H-Regel im Bundesland mit der größten Landesfläche, wie zuvor unter 3.2 geschildert, in eklatanter Weise gebremst wird, dann kann man sich mit guten Gründen auf den Standpunkt stellen, dass hieraus auch eine relevante Gefährdung des Grundrechts auf intertemporale Freiheitssicherung resultiert. Dies gilt jedenfalls dann, wenn man im Einklang mit einer in der rechtswissenschaftlichen Literatur vertretenen Ansicht davon ausgeht, dass das geltende Recht sich daran messen lassen muss, ob es zum Klimaschutz beiträgt oder diesen eher behindert. ${ }^{44}$ Hierfür spricht auch der Umstand, dass im Schrifttum schon wiederholt darauf hingewiesen wurde, dass sich die „dem Staat“ aufgegebene Pflicht zum Klimaschutz und zur Herstellung von Klimaneutralität auch an die Länder richte ${ }^{45}$ und dass diese Pflicht selbst bei Einzelfallentscheidungen der Exekutive, bei denen Gestaltungs-, Abwägungs- und Beurteilungsspielräume bestehen, zu berücksichtigen sei. ${ }^{46}$

\subsubsection{Die zu berücksichtigende Frage der Verteilungs- und Umweltgerechtigkeit}

Gegen eine relevante Gefährdung des Grundrechts auf intertemporale Freiheitssicherung könnte man freilich einwenden, dass die negativen Auswirkungen der 10-H-Regel auf den Klimaschutz nicht so problematisch sind, weil der Windenergieausbau aus Gründen der tendenziell größeren Windhöffigkeit ohnehin in deutlich größerem Umfang in Nord- als in Süddeutschland erfolgt. Und tatsächlich wird schon jetzt rund die Hälfte des Windstroms im Norden Deutschlands produziert. ${ }^{47}$

Indessen ist eine solche Sichtweise nicht nur aus einer umweltethischen, sondern mittlerweile auch aus einer verfassungsrechtlichen Perspektive abzulehnen, wenn man zusätzlich die soeben unter 4.2.2 angesprochene und im Klima-Beschluss des BVerfG angelegte Frage der Verteilungs- und Umweltgerechtigkeit in den Blick nimmt. Denn die großen Verbrauchsschwerpunkte liegen im Süden Deutschlands. Von daher sollte der erneuerbare Strom aus Gründen der Verteilungs- und Umweltgerechtigkeit verstärkt dort produziert werden, wo er gebraucht wird. ${ }^{48}$

Dies gilt umso mehr, als eine fortschreitende Konzentration der Stromproduktion im Norden dazu führt, dass sich der - ohnehin stockende - Netzausbaubedarf weiter vergrößert. Darüber hinaus steigen einerseits im Norden die Risiken von Netzüberlastungen, während andererseits im Süden die Gefahr von Versorgungsengpässen zunimmt. ${ }^{49}$ Schließlich ist das tendenzielle Nord-Süd-Gefälle bei genauerem Hinsehen keineswegs der höheren Windhöffigkeit des Nordens geschuldet. Denn in diesem Fall müsste etwa in Mecklenburg-Vorpommern mehr Windstrom produziert werden als etwa in Sachsen-Anhalt. Tatsächlich ist es aber umgekehrt. ${ }^{50}$

Aus diesen Gründen kann man, wie gesagt, mit guten Gründen die Ansicht vertreten, dass die 10-H-Regelung

39) Vgl. BVerfG, Beschl. v. 24.3.2021 - 1 BvR 2656/18, 1 BvR 78/29, 1 BvR 96/20, 1 BvR 288/20, Leitsatz 2 sowie Rdnr. 118 ff., 198, $208 \mathrm{ff}$.

40) Vgl. BMU, Klimaschutz in Zahlen - Fakten, Trends und Impulse deutscher Klimapolitik, Ausgabe 2021, S. 30.

41) Vgl. zum Ganzen $B M U$, Klimaschutz in Zahlen - Fakten, Trends und Impulse deutscher Klimapolitik, Ausgabe 2021, S. 33 f.

42) Vgl. zum Ganzen BMU, Klimaschutz in Zahlen - Fakten, Trends und Impulse deutscher Klimapolitik, Ausgabe 2021, S. $38 \mathrm{f}$.

43) Vgl. UBA, Auswirkungen von Mindestabständen zwischen Windenergieanlagen und Siedlungen - Auswertung im Rahmen der UBA-Studie „Flächenanalyse Windenergie an Land“, Positionspapier vom März 2019, Stand 5.10.2021, abrufbar unter https://www.umweltbundesamt.de/sites/default/files/medien/ 1410/publikationen/2019-03-20_pp_mindestabstaende-windenergieanlagen.pdf, S. 5.

44) Siehe Sinner, UPR 2021, $281 \mathrm{ff}$.

45) Vgl. etwa Schlacke, NVwZ 2021, 912, 917 sowie ausführlicher Ekardt/Heß/Wulff, EurUP 2021, 212, 222 f., die in diesem Kontext ausdrücklich auf die Problematik ausgreifender Abstandsregelungen für Windenergieanlagen hinweisen.

46) Vgl. etwa Faßbender, NJW 2021, 2085, Rdnr. 34 sowie ausführlicher Ekardt/Heß/Wulff, EurUP 2021, 212, $218 \mathrm{ff}$.

47) Vgl. etwa Stand 5.10.2021, abrufbar unter https://strom-report.de/windenergie.

48) Vgl. hierzu auch Köck, in: Hebeler/Hofmann/Proelß/Reiff, Jahrbuch des Umwelt- und Technikrechts 2017, S. 129, $133 \mathrm{ff}$.

49) Vgl. hierzu sowie allgemein zur Interdependenz von Netzausbau, Erzeugungsstruktur und Nachfrage Hermes, ZUR 2014, 259, 261.

50) Vgl. etwa https://strom-report.de/windenergie (zuletzt abgerufen am 8.12.2021) 
das neue Grundrecht auf intertemporale Freiheitssicherung in relevanter Weise gefährdet.

\subsubsection{Verbleibender Klärungsbedarf}

Es verbleibt hier freilich ein gewisser Klärungsbedarf. Denn in der rechtswissenschaftlichen Literatur wird ebenfalls darauf hingewiesen, dass das neue Grundrecht auf intertemporale Freiheitssicherung allein einen subjektiven Anspruch auf gerechte Verteilung von $\mathrm{CO}_{2}$-Budgets zwischen den Generationen vermittle und dass diese Verteilung in erster Linie Aufgabe des Bundesgesetzgebers sei. ${ }^{51}$ Vor diesem Hintergrund betonen manche die ,spezielle Konstellation“, die der Entscheidung des BVerfG zugrunde lag. Namentlich sei der ,intertemporale Zugriff ... auf den extrem hohen und in den faktischen Anteilen naturwissenschaftlich gehärteten Grad an Verknüpfung gegenwärtiger Maßnahmen (zugelassener Emissionsmengen nach dem KSG) mit zukünftigen staatlichen Beschränkungen geknüpft." Dementsprechend könne eine Übertragung auf diese spezielle Konstellation nur bei vergleichbar belastbaren, in allgemeineren politischen Zusammenhängen wohl regelmäßig nicht bestehenden Verdichtungen vorgenommen werden. ${ }^{52}$

Aus diesen Gründen bedarf die Frage, ob einzelne Vorschriften außerhalb des Bundes-Klimaschutzgesetzes (KSG), die so wie die 10-H-Regelung den Übergang zur vom BVerfG geforderten Klimaneutralität in durchaus erheblicher Weise hemmen, einer Überprüfung am Maßstab des neuen Grundrechts auf intertemporale Freiheitssicherung zugänglich sind, einer Klärung durch das BVerfG. Eine solche Klärung könnte etwa im Rahmen einer richterlichen Vorlage nach Art. 100 Abs. 1 S. 1 oder 2 GG erfolgen und wäre zeitnah durchaus wünschenswert, weil sie grundsätzlicher Natur ist.

\subsection{Vereinbarkeit der 10-H-Regelung mit den Grundrechten der Grundstückseigentümer und Anlagenbetreiber}

Letztlich kommt es hierauf aber nicht an, weil die 10-HRegelung jedenfalls in das grundrechtlich geschützte Recht der betroffenen Eigentümer eingreift, ihr Grundstück im Rahmen der Gesetze baulich zu nutzen (4.3.1). Ferner liegt auch ein relevanter Eingriff in die grundrechtlich geschützte Berufsfreiheit der Anlagenbetreiber vor (4.3.2). Deshalb stellt sich die bereits in der Vergangenheit kontrovers diskutierte Frage, ob dieser Grundrechtseingriff gerechtfertigt ist. Dabei wird zu zeigen sein, dass diese Frage vor dem Hintergrund des Klima-Beschlusses des BVerfG neu $\mathrm{zu}$ bewerten und letztlich $\mathrm{zu}$ verneinen ist (4.3.3).

\subsubsection{Die eigentumsbeschränkende Wirkung der 10-H-Regelung}

Eine der wesentlichen Inhalte der Eigentumsgarantie, die auf der Bundesebene durch Art. 14 Abs. 1 S. 1 GG und in Bayern durch Art. 103 Abs. 1 BayVerf gewährleistet wird, ist die sog. Baufreiheit als das Recht des Grundstückseigentümers, sein Grundstück grundsätzlich frei baulich nutzen zu können. Dies umfasst auch das Recht, auf dem eigenen Grundstück eine Windkraftanlage zu errichten oder die Errichtung zuzulassen. Demzufolge ist es im rechtswissenschaftlichen Schrifttum, soweit ersichtlich, unstreitig, dass die 10-H-Regelung einen Eingriff in das Eigentumsgrundrecht betroffener Grundstückseigentümer darstellt. ${ }^{53}$ Dies hat im Übrigen auch der BayVerfGH in seiner Entscheidung vom 9. 5.2016 so gesehen. ${ }^{54}$ Daher greift die 10-H-Regelung jedenfalls in das durch Art. 14 Abs. 1 S. 1 GG und Art. 103 Abs. 1 BayVerf geschützte Eigentumsgrundrecht von Grundstückseigentümern ein, die von der Regelung tatsächlich betroffen sind.

4.3.2 Die 10-H-Regelung als Eingriff in die Berufsfreiheit der Anlagenbetreiber

Nicht ganz eindeutig zu beantworten ist die Frage, ob die 10-H-Regelung eigenständig in die grundrechtlich ge- schützte Berufsfreiheit von Anlagenbetreibern eingreift, die zugleich Vorhabenträger sein können.

\subsubsection{Schutzbereich}

Hierzu müssen die Windenergieanlagen zunächst einmal berufsmäßig betrieben werden. Dies ist dann der Fall, wenn es sich um eine auf Dauer angelegte Tätigkeit zur Schaffung und Erhaltung einer Lebensgrundlage handelt. Dabei reicht es aus, wenn sich der Grundrechtsträger mit der Tätigkeit ein Auskommen verschafft. Es darf sich allerdings einerseits nicht um einen einmaligen Erwerbsakt handeln. Andererseits wird nicht nur die hauptberufliche Tätigkeit geschützt. Auch Zweitberufe, Nebentätigkeiten und Gelegenheitsarbeiten sind Berufe im Sinne von Art. 12 Abs. 1 GG. ${ }^{55}$ Diese (positiven) Voraussetzungen werden beim dauerhaften Betrieb von Windenergieanlagen für den allgemeinen Strombedarf in der Regel gegeben sein.

\subsubsection{Eingriff}

Sodann muss ein Eingriff in den Schutzbereich vorliegen. Dies ist allgemein jedenfalls dann zu bejahen, wenn eine gezielte Beschränkung des grundrechtlich geschützten Verhaltens vorliegt. Zu der durch Art. 12 Abs. 1 GG geschützten Berufsfreiheit zählt zwar nicht nur die berufliche Praxis selbst, sondern auch jede Tätigkeit, die mit der Berufsausübung zusammenhängt und dieser dient. Bezieht sich eine staatliche Maßnahme auf einen bestimmten Beruf wie zum Beispiel eine Erlaubnispflicht oder eine Regelung der Vergütung, so liegt nach der Rechtsprechung des BVerfG regelmäßig auch ein Eingriff in die Berufsfreiheit vor.

Weil es aber zahlreiche Regelungen gibt, die einen bestimmten Zweck verfolgen, die Berufsfreiheit aber nur mittelbar berühren, versucht die Rechtsprechung, und hier namentlich das BVerfG, in Übereinstimmung mit weiten Teilen des Schrifttums die relevanten Eingriffe auf solche mit „objektiv berufsregelnder Tendenz" zu begrenzen. ${ }^{56}$ Diese Rechtsprechung könnte möglicherweise dazu verleiten, eine baurechtliche Norm wie die 10-H-Regelung selbst dann nicht als einen Eingriff in die Berufsfreiheit von Anlagenbetreibern $\mathrm{zu}$ werten, wenn diese aus den zuvor unter 3 genannten Gründen faktisch in erheblicher Weise von der 10-H-Regelung bei der Ausübung ihrer gewerblichen Tätigkeiten betroffen sind.

Es ist aber zu beachten, dass diese Judikatur in der rechtswissenschaftlichen Literatur auch entschiedene Gegner gefunden hat. So wird zutreffend eingewandt, dass die Forderung nach einer berufsregelnden Tendenz im Wortlaut des Art. 12 Abs. 1 GG keinerlei Stütze findet. Es sei auch kein Grund ersichtlich, von der allgemeinen Eingriffsdogmatik abzuweichen, nach der grundsätzlich jede beschränkende Maßnahme ein Eingriff sein kann. Die möglicherweise geringere Eingriffsintensität könne auf der Rechtfertigungsebene verarbeitet werden. ${ }^{57}$

51) So etwa Schlacke, NVwZ 2021, 912, $916 \mathrm{f}$

52) So Eifert, JURA 2021, 1085, 1092.

53) Vgl. etwa Albrecht/Zschiegner, NVwZ 2015, 1093, 1094; Helmes/Klöcker, REE 2015, 81, 84; Winkler/Stückemann, UPR 2015, 516, 521.

54) Vgl. BayVerfGH, Entsch. v. 9. 5. 2016 - Vf. 14-VII-14, Vf. 3-VIII15, Vf. 4-VIII-15, Rdnr. 120.

55) Vgl. zum Ganzen hier nur Manssen, in: von Mangoldt/Klein/ Starck, Grundgesetz, 7. Aufl. 2018, Rdnr. 37 f. mit weiteren Nachweisen aus der Rechtsprechung.

56) Vgl. bspw. BVerfG Urt. v. 17.2.1998 - 1 BvF 1/91; zustimmend etwa Jarass, in: Jarass/Pieroth, Kommentar zum Grundgesetz, 16. Aufl. 2020, Art. 12 Rdnr. 15 ff.; Kingreen/Poscher, Grundrechte - Staatsrecht II, 34. Aufl. 2018, Rdnr. 951; Mann, in: Sachs, Kommentar zum Grundgesetz, 9. Aufl. 2021, Art. 12 Rdnr. 95-97.

57) So etwa die Kritik bei Ruffert, in: Epping/Hillgruber, Beck'scher Online-Kommentar zum Grundgesetz, Werkstand: 15.5.2021, Art. 12 Rdnr. 57; ebenfalls kritisch Manssen, in: von Mangoldt/ Klein/Starck, Grundgesetz, 7. Aufl. 2018, Rdnr. 75 ff. 
Angesichts dessen ist es zumindest geboten, die Forderung nach einer berufsregelnden Tendenz im Einzelfall großzügig zu handhaben, zumal auch das BVerfG betont, dass Regelungen ohne unmittelbaren Berufsbezug ebenfalls an Art. 12 Abs. 1 GG zu messen sind, ,wenn sie nach Entstehungsgeschichte und Inhalt im Schwerpunkt Tätigkeiten betreffen, die typischerweise beruflich ausgeübt werden." ${ }^{58}$ Letzteres ist, wie gesagt, beim dauerhaften Betrieb von Windenergieanlagen für den allgemeinen Strombedarf regelmäßig der Fall. Und an anderer Stelle heißt es in der Rechtsprechung, dass der besondere Freiheitsraum, den das Grundrecht der Berufsfreiheit sichern will, auch durch Vorschriften ohne primär berufsregelnde Zielrichtung berührt sein kann, wenn ihre tatsächlichen Auswirkungen zu einer Beeinträchtigung der freien Berufsausübung führen. ${ }^{59}$

Vor diesem Hintergrund wird im rechtswissenschaftlichen Schriftum im Ergebnis zu Recht die Ansicht vertreten, dass die 10-H-Regelung ebenfalls in die grundrechtlich geschützte Berufsfreiheit von Vorhabenträgern eingreift. ${ }^{60}$ Das Gleiche muss für die Betreiber von Windenergieanlagen gelten.

\subsubsection{Verhältnis zur Eigentumsgarantie}

Es ist aber zu klären, wie sich die Berufsfreiheit in diesem konkreten Fall zur Eigentumsgarantie verhält. Der BayVerfGH hat nämlich in seiner Entscheidung vom 9. 5.2016 den Standpunkt eingenommen, die 10-H-Regelung sei nicht am Grundrecht der allgemeinen Handlungsfreiheit (Art. 101 BayVerf) zu messen, dessen Schutz den beruflichen und wirtschaftlichen Bereich sowohl der Grundstückseigentümer als auch der - potenziellen - Betreiber von Windkraftanlagen umfasse. Denn dieses Grundrecht werde unter den vorliegenden Umständen durch das sachnähere Eigentumsgrundrecht verdrängt. Die Berufsfreiheit schütze den Erwerb, mithin die Betätigung selbst, während die Eigentumsgarantie das Erworbene schütze, also die Ergebnisse der Erwerbsbetätigung zum Gegenstand habe. ${ }^{61}$

Diese Ansicht vermag - zumal in ihrer Pauschalität - nicht zu überzeugen. Zwar entspricht die Aussage, die Berufsfreiheit schütze den Erwerb, während die Eigentumsgarantie das Erworbene schütze, also die Ergebnisse der Erwerbsbetätigung zum Gegenstand habe, im Grundsatz der Rechtsprechung des BVerfG und der herrschenden Lehre. ${ }^{62}$ Es ist aber ebenfalls weitgehend anerkannt, dass die Berufsfreiheit nicht stets durch die Eigentumsgarantie verdrängt wird. Die beiden Grundrechte haben vielmehr, je nach Sachverhalt, durchaus selbstständige Bedeutung mit der Folge, dass beide Grundrechte als Prüfungsmaßstab heranzuziehen sind. ${ }^{63}$ Dies ist im vorliegenden Zusammenhang jedenfalls dann anzunehmen, wenn Grundstückseigentümer und - potenzielle - Betreiber von Windkraftanlagen nicht zusammenfallen. Denn anderenfalls würden die Anlagenbetreiber, die nicht zugleich Grundstückseigentümer sind, verfassungsrechtlich schutzlos gestellt.

\subsubsection{Die neu zu stellende Frage}

nach der verfassungsrechtlichen Rechtfertigung

Deshalb stellt sich - so oder so - die bereits in der Vergangenheit kontrovers diskutierte Frage, ob die aus der 10-HRegelung resultierenden Grundrechtseingriffe gerechtfertigt sind.

\subsubsection{Relevante Änderungen der Prüfung durch den Klima-Beschluss}

Diese Frage ist vor dem Hintergrund des Klima-Beschlusses des BVerfG vom 24.3.2021 neu zu stellen und auch neu zu bewerten, da im Vorstehenden unter 4.1 und 2 schon deutlich geworden ist, dass sich aus dieser Entscheidung auch relevante Verschiebungen bei der Überprüfung von staatlichen Maßnahmen am Maßstab der Grundrechte ergeben.

Im Rahmen der verfassungsrechtlichen Rechtfertigung eines Grundrechtseingriffs ist üblicherweise zunächst zu prüfen, welchen Beschränkungsmöglichkeiten das betroffene Grundrecht unterliegt (sog. Schranken). Dies ist hier unproblematisch, da es sich bei der 10-H-Regelung um ein formelles Landesgesetz handelt, dessen formelle Verfassungsmäßigkeit im Folgenden unterstellt werden soll. Bei der Prüfung der materiellen Verfassungsmäßigkeit bildet der Verhältnismäßigkeitsgrundsatz den entscheidenden Maßstab, der in seinen Grundstrukturen allgemein anerkannt ist.

Dieser Verhältnismäßigkeitsgrundsatz findet nach dem Klima-Beschluss auch auf das neue Grundrecht auf intertemporale Freiheitssicherung Anwendung. ${ }^{64}$ Allerdings hat die Verhältnismäßigkeitsprüfung hier eine andere Struktur. ${ }^{65}$ Hier geht es hingegen um Eingriffe in das Eigentumsgrundrecht und in die Berufsfreiheit, so dass grundsätzlich der „klassische“ Verhältnismäßigkeitsgrundsatz heranzuziehen ist. Danach ist erstens zu fragen, ob die staatliche $\mathrm{Maßnahme}$ einen legitimen Zweck verfolgt. Zweitens ist $\mathrm{zu}$ ermitteln, ob die staatliche Maßnahme überhaupt geeignet ist, den Zweck zumindest zu fördern. Drittens muss die staatliche Maßnahme zur Erreichung des Zwecks erforderlich sein, was davon abhängt, ob es mildere Mittel gibt, die das Ziel ebenso effektiv fördern. Schließlich muss die staatliche Maßnahme viertens angemessen und zumutbar sein. Hier ist unter Berücksichtigung sämtlicher verfassungsrechtlich erheblicher Belange im Rahmen einer Abwägung zu klären, ob die Förderung des Ziels noch in einem angemessenen Verhältnis zu der damit verbundenen Freiheitseinbuße steht (sog. Verhältnismäßigkeit im engeren Sinne).

Geht es - so wie hier - um Fragen des Klimaschutzes, so wird dieser „,klassische“ Verhältnismäßigkeitsgrundsatz vor allem durch das sog. Klimaschutzgebot modifiziert, das im Klima-Beschluss aus Art. 20a GG hergeleitet wird und das der Bundesgesetzgeber - nach Ansicht des BVerfG verfassungsrechtlich maßgeblich - durch das Ziel konkretisiert hat, die Erwärmung der Erde bis 2050 auf deutlich unter $2^{\circ} \mathrm{C}$ und möglichst auf $1,5^{\circ} \mathrm{C}$ gegenüber dem vorindustriellen Niveau zu begrenzen. Insofern muss die „,dem Staat" aufgegebene Pflicht zum Klimaschutz auch auf die Herstellung von Klimaneutralität abzielen. ${ }^{66}$

Dieses so verstandene Klimaschutzgebot ist bei der Prüfung der Verhältnismäßigkeit jedenfalls auf der letzten Stufe und damit bei der Klärung der Angemessenheit und Zumutbarkeit zu beachten, die im Rahmen einer Abwägung zu erfolgen hat. Denn das BVerfG weist ausdrücklich allgemein darauf hin, dass die Vereinbarkeit mit dem so verstandenen Art. 20a GG „Voraussetzung für die verfassungsrechtliche Rechtfertigung staatlicher Eingriffe in Grundrechte" ist. ${ }^{67}$

Dabei ist nach dem Klima-Beschluss weiterhin zu beachten, dass das relative Gewicht des Klimaschutzgebots in der Abwägung bei fortschreitendem Klimawandel wei-

58) Vgl. etwa BVerfG, Urt. v. 17.2.1998 - 1 BvF 1/91.

59) So BVerfG, Urt. v. 17.2.1998 - $1 \mathrm{BvF} 1 / 91$.

60) Vgl. etwa Helmes/Klöcker, REE 2015, 81, 84.

61) Siehe BayVerfGH, Entsch. v. 9.5.2016 - Vf. 14-VII-14, Vf. 3-VIII-15, Vf. 4-VIII-15, Rdnr. 157.

62) Vgl. hier nur BVerfG, Beschl. v. 8.6.2010 - 1 BvR 2011/07, 1 BvR 2959/07 und Manssen, in: von Mangoldt/Klein/Starck, Grundgesetz, 7. Aufl. 2018, Rdnr. 289.

63) Vgl. hier nur BVerfG, Urt. v. 1.3.1979 - 1 BvR 532, 533/77, 419/78, $1 \mathrm{BvL} 21 / 78$ und Manssen, in: von Mangoldt/Klein/ Starck, Grundgesetz, 7. Aufl. 2018, Rdnr. 290.

64) Siehe BVerfG, Beschl. v. 24.3.2021 - 1 BvR 2656/18, 1 BvR 78/29, 1 BvR 96/20, 1 BvR 288/20, Rdnr. 188 ff., 192.

65) Siehe näher dazu Eifert, JURA 2021, 1085, 1093.

66) Vgl. BVerfG, Beschl. v. 24.3.2021 - 1 BvR 2656/18, 1 BvR 78/29, 1 BvR 96/20, 1 BvR 288/20, Leitsatz 2 sowie Rdnr. 118 ff., 198, $208 \mathrm{ff}$.

67) Siehe BVerfG, Beschl. v. 24.3.2021 - 1 BvR 2656/18, 1 BvR 78/29, 1 BvR 96/20, 1 BvR 288/20, Leitsatz 3. 
ter zunimmt. ${ }^{68}$ Schließlich betont das BVerfG im KlimaBeschluss, dass dem Gesetzgeber durch Art. 20a GG eine permanente Pflicht aufgegeben sei, das Umweltrecht den neuesten Entwicklungen und Erkenntnissen in der Wissenschaft anzupassen. ${ }^{69}$

\subsubsection{Fragwürdigkeit des Ziels der 10-H-Regelung}

Bevor eine solche Abwägung vorgenommen wird, muss aber geklärt werden, ob die 10-H-Regelung überhaupt einen legitimen Zweck verfolgt. Hier ist nach den Gesetzesmaterialien davon auszugehen, dass der Landesgesetzgeber mit dieser Regelung das Ziel verfolgt, die Akzeptanz von Windkraftanlagen in der Bevölkerung im Zusammenhang mit der Energiewende zu fördern. ${ }^{70}$ Dieses Ziel hat der BayVerfGH in seiner Entscheidung vom 9. 5.2016 zwar ohne nähere Auseinandersetzung gebilligt. ${ }^{71}$ Allerdings wird im Schrifttum zu Recht kritisch gefragt, ob die Förderung der Akzeptanz politischer Entscheidungen einen hinreichenden Eingriffszweck darstellt, weil es sich hierbei um ein ,äußerst weiches, nicht rationalisierbares Kriterium" handelt. ${ }^{72}$ Von daher ist schon das Ziel der 10-HRegelung fragwürdig.

\subsubsection{Fehlende Geeignetheit der 10-H-Regelung}

Sodann kann die pauschale Annahme des BayVerfGH in seiner Entscheidung vom 9.5.2016, dass die 10-H-Regelung geeignet sei, das Ziel der Akzeptanzsteigerung zu befördern, ${ }^{73}$ mittlerweile als widerlegt gelten. Hier ist zwar zu berücksichtigen, dass auch das BVerfG bei der Prüfung der Geeignetheit in der Regel recht großzügig verfährt. Das Gericht hat aber auch vereinzelt Vorschriften beanstandet, wenn es zwischen dem gesetzlichen Ziel und der Regelung keinen nachvollziehbaren Zusammenhang gab. ${ }^{74}$

Letzteres ist bei der 10-H-Regelung der Fall. So zeigen umweltpsychologische Untersuchungen, dass der unterstellte Zusammenhang zwischen Abstandsregelungen und der Akzeptanz in der Bevölkerung empirisch nicht nachweisbar ist. ${ }^{75}$ Und auch die Bundesregierung musste in ihrer Antwort vom 28.6.2018 auf eine Kleine Anfrage hin einräumen, dass kein Zusammenhang zwischen der ländereigenen Festlegung erhöhter Mindestabstände und einer höheren Akzeptanz empirisch nachgewiesen werden konnte. Für die Akzeptanz vor Ort spielten andere Faktoren eine zentrale Rolle, wie z.B. die Sichtbarkeit der Anlagen in der Landschaft, die Beteiligung und der Gestaltungsspielraum der Anwohner im Planungsprozess, inwiefern Anwohner finanziell beteiligt sind oder Wertschöpfung auch in der Region verbleibt. ${ }^{76}$

Diese Einschätzung wird durch folgenden schlichten Befund bestätigt. Knapp sieben Jahre nach Einführung der 10-H-Regelung zeigt sich in aller Deutlichkeit, dass diese Regelung die Akzeptanz von Windkraftanlagen in der Bevölkerung in keiner Weise gefördert hat. Denn in diesem Fall hätte die Windenergie wenigstens partiell an anderen Standorten in Bayern von der 10-H-Regelung profitieren müssen. Das ist aber, wie gezeigt, nicht festzustellen im Gegenteil. Vor diesem Hintergrund stellt das $U B A \mathrm{zu}$ Recht fest, dass pauschale Siedungsabstände wie die 10-HRegelung ,ein ungeeignetes Instrument zur Steigerung der Akzeptanz gegenüber der Windenergienutzung" darstellen. ${ }^{77}$

Bereits aus diesem Grund sollte der bayerische Gesetzgeber die 10-H-Regelung wieder aufheben. Hierfür spricht auch die im Klima-Beschluss betonte und aus Art. 20a GG destillierte Pflicht des Gesetzgebers, das Umweltrecht den neuesten Entwicklungen und Erkenntnissen in der Wissenschaft anzupassen. ${ }^{78}$ Denn diese Pflicht dürfte bei sachgerechtem Verständnis nicht nur für das eigentliche Umweltrecht, sondern gleichermaßen für sonstige klimaschutzrelevante Vorschriften gelten.

Schließlich ist mit Blick auf die Geeignetheit zu beachten, dass die 10-H-Regelung bei bestehenden Windenergieanlagen nicht nur die Akzeptanz nicht fördert, sondern die
Akzeptanz in der Bevölkerung sogar konterkarieren kann, weil sich die dort lebenden Personen benachteiligt fühlen könnten.

\subsubsection{Fehlende Erforderlichkeit der 10-H-Regelung}

Durchgreifenden Bedenken begegnet ferner die Erforderlichkeit der 10-H-Regelung. Diese hatte der BayVerfGH in seiner Entscheidung vom 9.5.2016 noch mit dem Argument bejaht, dass die geringeren Abstandserfordernisse, die sich aus dem Immissionsschutzrecht oder aus dem bauplanungsrechtlichen Gebot der Rücksichtnahme ergeben, nicht zu berücksichtigen seien. Denn Bezugspunkt der Erforderlichkeit sei das vom Landesgesetzgeber angestrebte Ziel einer ,,allgemeinen“ Akzeptanzförderung durch einen „großzügigen" Mindestabstand generell-abstrakter Art, nicht aber die (bloße) Abwehr unzumutbarer Einwirkungen auf die Nachbarschaft im Einzelfall. ${ }^{79}$

Diese Argumentation vermag schon deshalb nicht (mehr) $\mathrm{zu}$ überzeugen, weil sich gezeigt hat, dass bereits das Ziel einer ,allgemeinen“ Akzeptanzförderung fragwürdig ist und dass die 10-H-Regelung zur Förderung dieses Ziels schlicht nicht geeignet ist. Sodann wurde bereits im Vorstehenden unter 2.1 darauf hingewiesen, dass das geltende Recht und die Rechtsprechung bereits vor Einführung der 10-H-Regelung dem berechtigten Anliegen des Schutzes der Wohnbebauung vor einer „optisch bedrängenden Wirkung" Rechnung getragen haben. Auch hierdurch wird die Erforderlichkeit der 10-H-Regelung in Frage gestellt. ${ }^{80}$ Zudem ist die Beteiligung der Anwohner in einem anlassbezogenen Projektplanungsprozess, in dem auch geklärt werden könnte, inwiefern Anwohner finanziell beteiligt werden könnten, nicht nur ein milderes und gleich geeignetes, sondern nach der zuvor erwähnten Antwort der Bundesregierung vom 28.6.2018 sogar ein deutlich besser

68) Vgl. BVerfG, Beschl. v. 24.3.2021 - 1 BvR 2656/18, 1 BvR 78/29, 1 BvR 96/20, 1 BvR 288/20, Leitsatz 2a sowie Rdnr. 120, 185, 198.

69) Vgl. BVerfG, Beschl. v. 24.3.2021 - 1 BvR 2656/18, 1 BvR 78/29, 1 BvR 96/20, 1 BvR 288/20, Rdnr. 212.

70) Vgl. Bayerischer Landtag, Drs. 17/2137, S. 1, 6.

71) Vgl. BayVerfGH, Entsch. v. 9.5.2016 - Vf. 14-VII-14, Vf. 3-VIII15, Vf. 4-VIII-15, Rdnr. 148.

72) Siehe Winkler/Stückemann, UPR 2016, 516, 521; vgl. auch die kritischen Hinweise bei Lege, NVwZ 2019, 1000, $1002 \mathrm{f}$.

73) Vgl. BayVerfGH, Entsch. v. 9.5.2016 - Vf. 14-VII-14, Vf. 3-VIII15, Vf. 4-VIII-15, Rdnr. 148.

74) Vgl. bspw. BVerfG, Beschl. v. 5.11.1980 - 1 BvR 290/78; weitere Nachweise bei Sachs, in: Sachs, Kommentar zum Grundgesetz, 9. Aufl. 2021, Art. 20 Rdnr. 150.

75) Siehe Hoen/Firestone/Rand/Elliot/Hübner/Pohl/Wiser/Lantz/Haac/ Kaliski, Attitudes of U.S. Wind Turbine Neighbors: Analysis of a Nationwide Survey, Energy Policy 134, 2019, 110981, Stand 24.10.2021, abrufbar unter https://www.sciencedirect.com/ science/article/pii/S0301421519305683/pdfft? md5=f6ff6226 7f6bafdb0cc113820f789fed\&pid=1-s2.0-S0301421519305683main.pdf; Hübner/Pohl, Mehr Abstand - mehr Akzeptanz? Ein umweltpsychologischer Studienvergleich, Fachagentur Windenergie an Land, 2015, Stand 5.10.2021, abrufbar unter https:// filer.itz.uni-halle.de/dl//698/pub/Huebner_Pohl_2015.pdf.

76) Vgl. BT-Drs. 19/3053, S. 1.

77) Vgl. Vgl. UBA, Auswirkungen von Mindestabständen zwischen Windenergieanlagen und Siedlungen - Auswertung im Rahmen der UBA-Studie „Flächenanalyse Windenergie an Land“, Positionspapier vom März 2019, Stand 5.10.2021, abrufbar unter https://www.umweltbundesamt.de/sites/default/files/medien/ 1410/publikationen/2019-03-20_pp_mindestabstaende-windenergieanlagen.pdf, S. 21 (Hervorhebung im Original).

78) Vgl. BVerfG, Beschl. v. 24.3.2021 - 1 BvR 2656/18, 1 BvR 78/29, 1 BvR 96/20, 1 BvR 288/20, Rdnr. 212.

79) Vgl. BayVerfGH, Entsch. v. 9. 5.2016 - Vf. 14-VII-14, Vf. 3-VIII15, Vf. 4-VIII-15, Rdnr. 148.

80) Vgl. näher dazu Albrecht/Zschiegner, NVwZ 2015, 1093, 1095 f.; Helmes/Klöcker, REE 2015, 81, 84. 
geeignetes Instrument zur Förderung der Akzeptanz von Windenergieanlagen. ${ }^{81}$

\subsubsection{Unangemessenheit der 10-H-Regelung}

Nimmt man vor diesem Hintergrund eine umfassende Abwägung unter Berücksichtigung sämtlicher verfassungsrechtlich erheblicher Belange vor, so erweist sich die 10-H-Regelung darüber hinaus im Ergebnis als nicht mehr angemessen und damit unzumutbar. Hieran vermögen die entgegenstehenden Feststellungen des BayVerfGH nichts zu ändern, weil die Entscheidung auch insoweit überprüfungsbedürftig ist. ${ }^{82}$ Denn auch hier ist zu berücksichtigen, dass erstens bereits das mit der Regelung verfolgte Ziel fragwürdig ist. Aber selbst wenn man die allgemeine Förderung der Akzeptanz vor Ort als legitimes Ziel einstuft, sprechen sämtliche vorliegende Erkenntnisse dafür, dass die 10-H-Regelung zweitens schon abstrakt nicht geeignet ist, dieses Ziel zu fördern. Bereits aus diesen Gründen ist der bayerische Gesetzgeber nach den Hinweisen des BVerfG im Klima-Beschluss drittens gehalten, die 10-H-Regelung an die neueren Entwicklungen und Erkenntnisse in der Wissenschaft anzupassen.

Das zuletzt Gesagte gilt viertens auch mit Blick auf die oben unter 2.2 skizzierten neueren Entwicklungen, die in aller Deutlichkeit die negativen rechtlichen und tatsächlichen Folgen der 10-H-Regelung für den Ausbau der Windenergie in Bayern belegen. Schon von daher vermag fünftens der Hinweis des BayVerfGH auf die verbleibende Möglichkeit einer Zulassung von Windenergieanlagen auf der Grundlage von $₫ 35$ Abs. 2 BauGB nicht (mehr) zu überzeugen. ${ }^{83}$ Es hat sich vielmehr insoweit klar die oben unter 3.1 vertretene These bestätigt, dass eine solche Genehmigung auf der Grundlage von $₫ 35$ Abs. 2 BauGB schon aus rechtlichen Gründen kaum in Betracht kommt. ${ }^{84}$

Das zeigt aber wiederum, dass der BayVerfGH sechstens das Gewicht der Eigentumsbeeinträchtigung auf Seiten der betroffenen Grundstückseigentümer nicht zutreffend gewichtet hat. Diese Fehlgewichtung wird dadurch noch weiter verstärkt, dass der Verfassungsgerichtshof siebtens die negativen Wirkungen der 10-H-Regelung auf die Möglichkeiten eines Repowering nicht näher gewürdigt hat, was in besonderem Maße unverständlich erscheint, weil die hiervon betroffenen Anwohner sich zumeist schon an die Windenergieanlage(n) gewöhnt haben, so dass Akzeptanzprobleme, wenn überhaupt, allenfalls eine untergeordnete Rolle spielen.

Hinzu kommt, dass der BayVerfGH achtens in fragwürdiger Weise die möglichen Eingriffe in die Berufsfreiheit von Anlagenbetreibern überhaupt nicht bei seiner Abwägung berücksichtigt hat (siehe näher dazu oben unter 4.3.3). Umgekehrt sind die genannten negativen Folgen für den Ausbau der Windenergie seit dem Klima-Beschluss neuntens mit einem erhöhten verfassungsrechtlichen Gewicht in die Abwägung einzustellen, weil die 10-H-Regelung den Übergang zur vom BVerfG geforderten Klimaneutralität in durchaus erheblicher Weise hemmt (siehe näher dazu oben unter 4.2.3), ohne dass dem entsprechende Vorteile gegenüberstehen. Das erscheint mit Blick auf Art. 20a GG bedenklich. Dieses verfassungsrechtliche Gewicht nimmt nach den Feststellungen des BVerfG im Klima-Beschluss zehntens bei fortschreitendem Klimawandel weiter zu.

Aus diesen Gründen ist die 10-H-Regelung jedenfalls nicht mehr verhältnismäßig im engeren Sinne, weil ihre erheblichen Nachteile für die Grundrechtsbetroffenen und auch für den Klimaschutz gegenüber den - ohnehin fragwürdigen - Vorteilen deutlich überwiegen.

\section{Zusammenfassung}

Damit ist zusammenfassend Folgendes festzuhalten:

1. Jede Person, die sich fachlich fundiert mit dem Ausbau der Windenergie an Land befasst, ist sich darüber im Kla- ren, dass die Flächenverfügbarkeit hier das größte Problem darstellt. Dessen ungeachtet enthält die Bayerische Bauordnung seit dem Jahr 2014 eine sog. 10-H-Regelung, die dazu führt, dass Windenergieanlagen in Bayern grundsätzlich nur noch dann gebaut werden dürfen, wenn der $\mathrm{Ab}-$ stand zur nächstgelegenen Wohnbebauung mindestens dem Zehnfachen der Anlagenhöhe $(\mathrm{H})$ entspricht. Dementsprechend muss der Abstand zur nächstgelegenen Wohnbebauung bei modernen Windenergieanlagen, die meist eine Höhe von $200 \mathrm{~m}$ oder mehr aufweisen, mindestens $2000 \mathrm{~m}$, also mindestens $2 \mathrm{~km}$ betragen.

2. Die 10-H-Regelung führt nach einem vom $U B A$ in Auftrag gegebenen Forschungsvorhaben zu einer Reduzierung der Fläche für die Windenergienutzung um 85 bis $97 \%$. In der Folge ist der Windenergieausbau in Bayern nahezu vollkommen zum Erliegen gekommen. Ferner wirkt sich die 10-H-Regelung überaus negativ auf die Möglichkeiten eines Repowering, also die Ersetzung vorhandener Windenergieanlagen durch neue leistungsfähigere Anlagen aus.

3. Wenngleich derartige negative Auswirkungen von manchen frühzeitig vorhergesagt wurden, hat der BayVerfGH die 10-H-Regelung im Jahre 2016 im Wesentlichen aus der Perspektive der Verfassung des Freistaates Bayern gebilligt.

4. Angesichts der zentralen Bedeutung des Windenergieausbaus für die Energiewende und für den Klimaschutz in Deutschland insgesamt bedarf die 10-H-Regelung jedoch einer verfassungsrechtlichen Neubewertung, da der sog. Klima-Beschluss des BVerfG vom 24.3.2021 einige verfassungsrechtliche Neuerungen mit sich gebracht hat, die zu einer signifikanten Stärkung des Klimaschutzes führen.

5. Eine der zentralen Neuerungen des Klima-Beschlusses ist die Aussage, dass das Grundgesetz unter bestimmten Voraussetzungen zur Sicherung grundrechtsgeschützter Freiheit über die Zeit und zur verhältnismäßigen Verteilung von Freiheitschancen über die Generationen verpflichte. Dahinter steht unter anderem die Annahme, dass Freiheit auch ein verteilungsfähiges Gut ist, was sodann Fragen der Umweltgerechtigkeit nach sich zieht. Aus diesen und anderen Gründen sollte der erneuerbare Strom anders als bisher - verstärkt dort produziert werden, wo er gebraucht wird: im Süden Deutschlands. Allerdings bedarf die Frage, ob einzelne Vorschriften außerhalb des Bundes-Klimaschutzgesetzes, die so wie die 10-H-Regelung den Übergang zur geforderten Klimaneutralität in durchaus erheblicher Weise hemmen, einer Überprüfung am Maßstab des neuen Grundrechts auf intertemporale Freiheitssicherung zugänglich sind, einer Klärung durch das BVerfG.

6. Letztlich kommt es hierauf aber nicht an, weil die 10-H-Regelung jedenfalls in das grundrechtlich geschützte Recht der betroffenen Eigentümer eingreift, ihr Grundstück im Rahmen der Gesetze baulich zu nutzen. Ferner liegt auch ein relevanter Eingriff in die grundrechtlich geschützte Berufsfreiheit der Anlagenbetreiber vor. Deshalb stellt sich die bereits in der Vergangenheit kontrovers diskutierte Frage, ob dieser Grundrechtseingriff gerechtfertigt ist.

7. Diese Frage ist ebenfalls neu zu stellen und auch neu zu bewerten, weil das BVerfG im Klima-Beschluss aus Art. 20a GG ein sog. Klimaschutzgebot hergeleitet hat,

81) Vgl. BT-Drs. 19/3053, S. 1; siehe zur Erforderlichkeit auch die Kritik bei Winkler/Stückemann, UPR 2016, 516, 522.

82) Siehe zur Verhältnismäßigkeit im engeren Sinne BayVerfGH, Entsch. v. 9.5.2016 - Vf. 14-VII-14, Vf. 3-VIII-15, Vf. 4-VIII15, Rdnr. 149.

83) Siehe BayVerfGH, Entsch. v. 9.5.2016 - Vf. 14-VII-14, Vf. 3-VIII-15, Vf. 4-VIII-15, Rdnr. 149.

84) Siehe auch die diesbezügliche Kritik an der Entscheidung des BayVerfGH bei Winkler/Stückemann, UPR 2016, 516, 522. 
das ,den Staat“, und damit Bund und Länder, aber auch Kommunen zur Herstellung von Klimaneutralität verpflichtet. Dieses Klimaschutzgebot ist bei Eingriffen in das Eigentumsgrundrecht und in die Berufsfreiheit bei der Prüfung der Verhältnismäßigkeit zu berücksichtigen. Dabei ist nach dem Klima-Beschluss weiterhin zu beachten, dass das relative Gewicht des Klimaschutzgebots in der Abwägung bei fortschreitendem Klimawandel weiter zunimmt. Schließlich betont das Verfassungsgericht im Klima-Beschluss, dass dem Gesetzgeber durch Art. 20a GG eine permanente Pflicht aufgegeben sei, das Umweltrecht den neuesten Entwicklungen und Erkenntnissen in der Wissenschaft anzupassen.

8. Bei der Prüfung der Verhältnismäßigkeit der 10-HRegelung ist bereits zweifelhaft, ob die Förderung der Akzeptanz des Ausbaus der Windenergie einen hinreichenden Eingriffszweck darstellt, weil es sich hierbei um ein äußerst weiches und nicht rationalisierbares Kriterium handelt.

9. Selbst wenn man das anders sieht, kann jedenfalls die pauschale Annahme des BayVerfGH, dass die 10-H-Regelung geeignet sei, das Ziel der Akzeptanzsteigerung zu befördern, mittlerweile als widerlegt gelten. Vor diesem Hintergrund stellt das $U B A$ zu Recht fest, dass pauschale Siedungsabstände wie die 10-H-Regelung schlicht ein ungeeignetes Instrument zur Steigerung der Akzeptanz gegenüber der Windenergienutzung darstellen.

10. Durchgreifenden Bedenken begegnet ferner die verfassungsrechtlich gebotene Erforderlichkeit der 10-H-Regelung, weil die Beteiligung der Anwohner in einem anlassbezogenen Projektplanungsprozess, in dem auch geklärt werden könnte, inwiefern Anwohner finanziell beteiligt werden könnten, nicht nur ein milderes und gleich geeignetes, sondern ein deutlich besser geeignetes Instrument zur Förderung der Akzeptanz von Windenergieanlagen ist.
11. Nimmt man vor diesem Hintergrund eine umfassende Abwägung unter Berücksichtigung sämtlicher verfassungsrechtlich erheblicher Belange vor, so erweist sich, dass die 10-H-Regelung jedenfalls nicht (mehr) angemessen und damit nicht verhältnismäßig im engeren Sinne ist, weil ihre erheblichen Nachteile für die Grundrechtsbetroffenen und für den Klimaschutz gegenüber den - ohnehin fragwürdigen - Vorteilen deutlich überwiegen.

12. Damit ist als Gesamtergebnis festzuhalten, dass die 10-H-Regelung verfassungsrechtlich nicht mehr haltbar ist, wenn man in gebührender Weise die Aussagen des BVerfG im Klima-Beschluss sowie - damit zusammen-hängend die neuesten Entwicklungen und Erkenntnisse zu den Wirkungen der 10-H-Regelung berücksichtigt.

Open Access. Dieser Artikel wird unter der Creative Commons Namensnennung 4.0 International Lizenz veröffentlicht, welche die Nutzung, Vervielfältigung, Bearbeitung, Verbreitung und Wiedergabe in jeglichem Medium und Format erlaubt, sofern Sie den/die ursprünglichen Autor(en) und die Quelle ordnungsgemäß nennen, einen Link zur Creative Commons Lizenz beifügen und angeben, ob Änderungen vorgenommen wurden.

Die in diesem Artikel enthaltenen Bilder und sonstiges Drittmaterial unterliegen ebenfalls der genannten Creative Commons Lizenz, sofern sich aus der Abbildungslegende nichts anderes ergibt. Sofern das betreffende Material nicht unter der genannten Creative Commons Lizenz steht und die betreffende Handlung nicht nach gesetzlichen Vorschriften erlaubt ist, ist für die oben aufgeführten Weiterverwendungen des Materials die Einwilligung des jeweiligen Rechteinhabers einzuholen.

Weitere Details zur Lizenz entnehmen Sie bitte der Lizenzinformation auf http://creativecommons.org/licenses/by/4.0/deed.de.

Open Access funding enabled and organized by Projekt DEAL.

\title{
Zur Rolle artenschutzrechtlicher Ausnahmen im Rahmen der raumordnerischen Konzentrationszonenplanung für die Windenergie: Ausnahmevoraussetzungen nach §45 Abs. 7 S. 1 BNatSchG*
}

\author{
Stephan Wagner
}

() Der/die Autor(en) 2021. Dieser Artikel ist eine Open-Access-Publikation.

Angesichts des ungebrochenen Ausbaubedarfs der Windenergie und zunehmend knapper werdender Standorte rückt die artenschutzrechtliche Ausnahme nach $\int 45$ Abs. 7 BNatSchG mehr und mehr in den Fokus der Raumplanung. Im vorliegenden Beitrag wird die in Rechtsprechung und Literatur aktuell hochumstrittene Frage untersucht, ob für die Errichtung von Windkraftanlagen überhaupt ein Ausnahmegrund gemäß 545 Abs. 7 S. 1 BNatSchG geltend gemacht werden kann. In einem demnächst erscheinenden zusätzlichen Beitrag werden sodann die weiteren Ausnahmevoraussetzungen nach $\int 45$ Abs. 7 S. 2 BNatSch G sowie die für Planungspraxis und Projektträger nicht unwesentliche Frage erörtert, ob und inwiefern die Ausnahmeerteilung durch die Naturschutzbehörden

Dr. Stephan Wagner, Akademischer Rat a.Z am Institut für OOffentliches Recht und Politik, Westfälische Wilhelms-Universität Münster, Münster, Deutschland an eine gewisse Regelhaftigkeit und damit Vorhersehbarkeit gebunden werden kann.

\section{Einleitung}

\subsection{Einführung und Problemstellung}

Die Errichtung von Windkraftanlagen verwirklicht an vielen an sich für eine Projektierung in Frage kommenden Standorten das artenschutzrechtliche Tötungsverbot

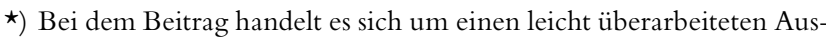
schnitt eines Rechtsgutachtens zum Pilotprojekt Zukunft naturverträgliche Windenergie in Baden-Württemberg, das der Verfasser im Auftrag des NABU (Naturschutzbund Deutschland) Landesverband Baden-Württemberg e. V. erstellt hat. Der Beitrag gibt ausschließlich die wissenschaftliche Meinung des Verfassers wieder. 